This item was submitted to Loughborough's Research Repository by the author.

Items in Figshare are protected by copyright, with all rights reserved, unless otherwise indicated.

\title{
A robust hybrid feedback active noise cancellation headset
}

PLEASE CITE THE PUBLISHED VERSION

http://dx.doi.org/10.1109/TSA.2005.848878

PUBLISHER

(c) IEEE

VERSION

AM (Accepted Manuscript)

LICENCE

CC BY-NC-ND 4.0

REPOSITORY RECORD

Song, Ying, Yu Gong, and Sen M. Kuo. 2019. "A Robust Hybrid Feedback Active Noise Cancellation Headset". figshare. https://hdl.handle.net/2134/25679. 


\title{
A Robust Hybrid Feedback Active Noise Cancellation Headset
}

\author{
Ying Song, Yu Gong, and Sen M. Kuo, Senior Member, IEEE
}

\begin{abstract}
This paper investigates the robustness of a hybrid analog/digital feedback active noise cancellation (ANC) headset system. The digital ANC systems with the filtered-x least-mean-square (FXLMS) algorithm require accurate estimation of the secondary path for the stability and convergence of the algorithm. This demands a great challenge for the ANC headset design because the secondary path may fluctuate dramatically such as when the user adjusts the position of the ear-cup. In this paper, we analytically show that adding an analog feedback loop into the digital ANC systems can effectively reduce the plant fluctuation, thus achieving a more robust system. The method for designing the analog controller is highlighted. A practical hybrid analog/digital feedback ANC headset has been built and used to conduct experiments, and the experimental results show that the hybrid headset system is more robust under large plant fluctuation, and has achieved satisfactory noise cancellation for both narrowband and broadband noises.
\end{abstract}

Index Terms-Active noise cancellation (ANC), feedback control, filtered-x least-mean-square (FXLMS), headset.

\section{INTRODUCTION}

A $\mathrm{N}$ active noise cancellation (ANC) headset can be used in a highly noisy environment for protecting human hearing ability and improving communication quality. It can be implemented using either feedforward or feedback technique. The feedforward ANC headset is effective in the open space with single noise source and in the enclosed space with weak reflections. However, the environmental noise is either omni-directional or at least multi-directional in many practical applications, where the feedback ANC headset is preferred [1], [2].

The first feedback ANC headset illustrated in Fig. 1 was proposed by Olson and May [3] in 1953, where the loudspeaker (SPK), microphone (MIC), and controller were used to form the feedback loop for canceling the undesired noise. Other early development of the ANC headset also adopted this structure with an analog controller [4]. This analog feedback ANC headset achieves satisfactory broadband noise reduction due to the short time delay of analog components used in the controller [3]-[6], but it is difficult to reduce narrowband noises such as those generated by turbofans, helicopters, airplanes,

Manuscript received May 13, 2003; revised April 20, 2004. The Associate Editor coordinating the review of this manuscript and approving it for publication was Dr. Shoji Makino.

Y. Song is with the Institute for Infocomm Research, Singapore 119613 (e-mail: sying@i2r.a-star.edu.sg).

Y. Gong is with the Queen's University of Belfast, Belfast, U.K. (e-mail: y.gong@ee.qub.ac.uk).

S. M. Kuo is with the Department of Electrical Engineering, Northern Illinois University, Dekalb, IL 60115 USA (e-mail: kuo@ ceet.niu.edu).

Digital Object Identifier 10.1109/TSA.2005.848878

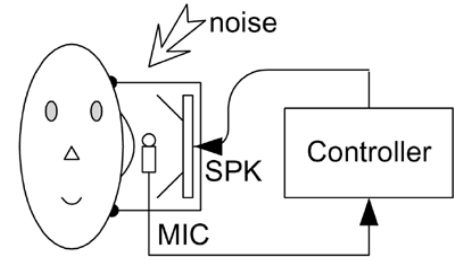

Fig. 1. Feedback ANC headset.

and etc. Furthermore, the headset with the analog controller is unable to track the environmental changes such as adjusting the ear-cup positions. The digital ANC system using an adaptive filter is thus preferred for headset applications because it can effectively track noise variations.

The digital ANC system usually uses the FXLMS algorithm [7] to update its adaptive filter coefficients. The FXLMS algorithm requires accurate estimation of the secondary path (from the output of the adaptive filter to the output of error microphone) for the convergence and stability of the system [2]. It has been shown that the sufficient condition for the stability of the feedforward FXLMS algorithm is the maximum phase estimation error of the secondary path must be within $90^{\circ}$ [8], and there is no simple relationship between error and stability within this range [9]. A counterpart of the $90^{\circ}$ condition for the feedback FXLMS algorithm has also been obtained by Sakai et al. in [10], [11], where it shows that the stability of the feedback FXLMS depends on the spectral properties of the secondary path and the targeting noise. It is generally recognized that feedback FXLMS algorithm has more strict stability condition than its feedforward counterpart [2]. Without a priori knowledge of the spectral properties of the secondary path and the noise, the $90^{\circ}$ condition can be used as an indicator of the feedback FXLMS stability. Violating the $90^{\circ}$ condition in feedback FXLMS algorithm may lead to small stability margin, though the algorithm might still be stable at some frequencies.

The accurate phase estimation required by the FXLMS algorithm brings great challenge to the ANC headset design because the physical plant may vary dramatically when the wearer adjusts the ear-cup positions, and the worst case is that the wearer temporarily pulls the ear-cup away from the ear. This drastic change makes the ANC system difficult to track plant fluctuation, and may cause the algorithm to diverge. The stability problem exists for both feedforward and feedback ANC headsets, but is more serious in the feedback ANC systems because the stability of the feedback loop must also be ensured. For example, when the ear-cup is pulled away from the ear, the feedback loop in the feedback ANC system will become 


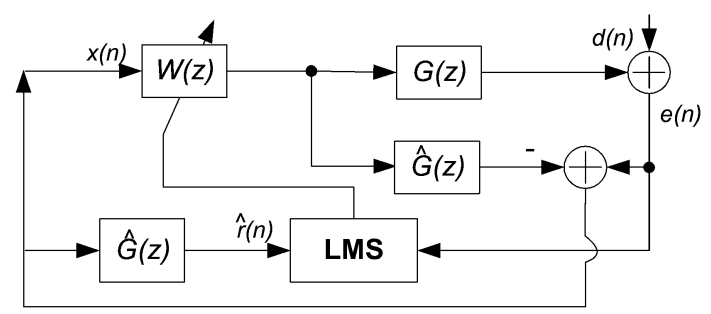

Fig. 2. Block diagram of the adaptive feedback ANC system with the FXLMS algorithm.

unstable, which will further diverge the FXLMS algorithm. Unless we reset the coefficients of the adaptive filter, the feedback ANC system will still be unstable after the ear-cup is put back to cover the ear. In contrast, the feedforward ANC headset without feedback loop does not have this problem, and the system will automatically re-converge once the unstable factors are removed. The detailed discussion of the feedback loop stability can be found in [2], and the application of adaptive feedback ANC for audio and communication headsets is reported in [12].

In this paper, we will analytically show that adding an analog feedback loop into digital ANC systems can effectively reduce the plant fluctuation and improve the stability of the FXLMS algorithm. It is known that using both analog and digital controllers has the advantage of canceling both broadband and narrowband noises [2]. In this paper, we will show that the hybrid analog/digital arrangement improves noise reduction and increases the stability margin. An analog controller that is tuned for maximizing noise reduction may not be stable. Therefore, most recent analog controller designs [13]-[16] must be modified for hybrid ANC systems. A method for designing a robust analog controller by maximizing the noise attenuation subject to several constraints [13] will be modified in this paper by adding an extra constraint to improve the stability of the FXLMS algorithm. Finally, we build a hybrid analog/digital feedback ANC headset for real-time experiments to verify the analysis results.

The rest of this paper is organized as follows: Section II addresses the problem of the plant fluctuation when the positions of the ear-cups are adjusted; Section III analyzes the reduction of plant fluctuation by adding the analog feedback controller, and briefly describes the method to design the analog controller. Finally, real-time experiment results of the hybrid analog/digital feedback ANC headset are given in Section IV.

\section{Problem Statement}

The block diagram of the FXLMS algorithm for the adaptive feedback ANC system is illustrated in Fig. 2, where $d(n)$ is the noise to be canceled, $x(n)$ is the synthesized reference signal, $e(n)$ is the residual noise measured by the error microphone, $W(z)$ is the transfer function of the digital controller, $G(z)$ is the transfer function of the secondary path, and $\hat{G}(z)$ is the estimate of $G(z)$. The feedforward ANC has similar structure except that its reference input $x(n)$ is sensed by a reference microphone instead of synthesizing the reference signal internally [1], [2].
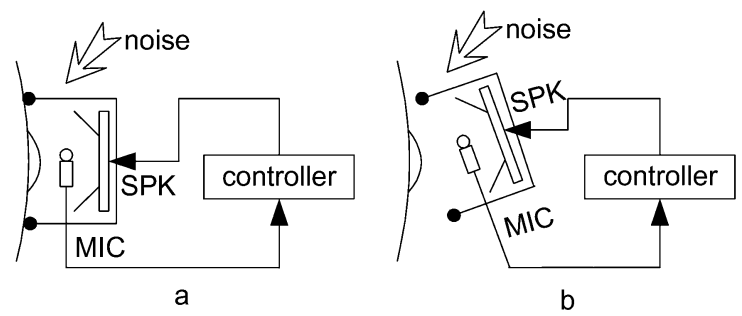

Fig. 3. Two different ear-cup positions.

The FXLMS algorithm is expressed as

$$
\mathbf{w}(n+1)=\mathbf{w}(n)-\mu e(n) \hat{\mathbf{r}}(n)
$$

where $\mu$ is the adaptation step-size, $\mathbf{w}(n)$ is the weight vector at time $n, e(n)=d(n)+\mathbf{w}^{\mathrm{T}}(n) \mathbf{r}(n), r(n)$ and $\hat{r}(n)$ are obtained from the convolutions $r(n)=g(n) * x(n)$ and $\hat{r}(n)=$ $\hat{g}(n) * x(n)$, respectively, $g(n)$ and $\hat{g}(n)$ are the impulse responses of $G(z)$ and $\hat{G}(z)$, respectively. As was stated in the Introduction, without a priori knowledge of the spectral properties of the secondary path and the noise, we should have $|\theta|<90^{\circ}$ (or $\cos \theta>0$ ) to ensure the stability of the FXLMS algorithm, where $\theta$ is the phase difference between the frequency response of $G(z)$ and $\hat{G}(z)$.

In general, the secondary path $G(z)$ can be estimated using either on-line or off-line techniques. The on-line estimation algorithm usually uses an additional adaptive filter to track the plant fluctuations in real time by injecting the identification noise (usually a low-level white noise) into the system [1], [2]. This technique is allowed for some ANC applications such as electronic mufflers. However, the need for extra modeling noise makes the on-line modeling method inappropriate for headset applications. Thus ANC headsets usually estimate the secondary path using the off-line technique [1] before the on-line noise cancellation. Under the normal circumstance that the ear-cup position is fixed, off-line modeling is adequate. However, in the case that the wearer adjusts the position of the ear-cup, $G(z)$ may vary drastically so that the system becomes unstable. We investigated the worst phase fluctuation when the ear-cup is totally pulled away from the ear, i.e., the position of ear-cup is changed from the position 1 to the position 2 as shown in Fig. 3.

In practical headset applications, the secondary path is normally estimated when the ear-cup is placed at the position 1 that covers the ear properly. Assuming that the estimation is accurate enough, we have $\hat{G}(z) \approx G(z)$ at position 1 . When the ear-cup is pulled away from the ear (at the position 2), $G(z)$ has changed, but the algorithm still uses the same estimation $\hat{G}(z)$ measured from position 1. This increases the phase estimation error that may be large enough for the FXLMS algorithm to diverge. To reveal the phase error, we estimate the phase responses at positions 1 and 2. Assuming our measurements are accurate, the phase estimation error at position 2 (shown in Fig. 4) equals the difference between the phases measured at positions 1 and 2 . It clearly shows that for frequencies higher than $400 \mathrm{~Hz}$, the phase error exceeds $90^{\circ}$, which violates the stability condition for the FXLMS algorithm. This is the reason the headset becomes unstable when the ear-cup is pulled away from the ear. 


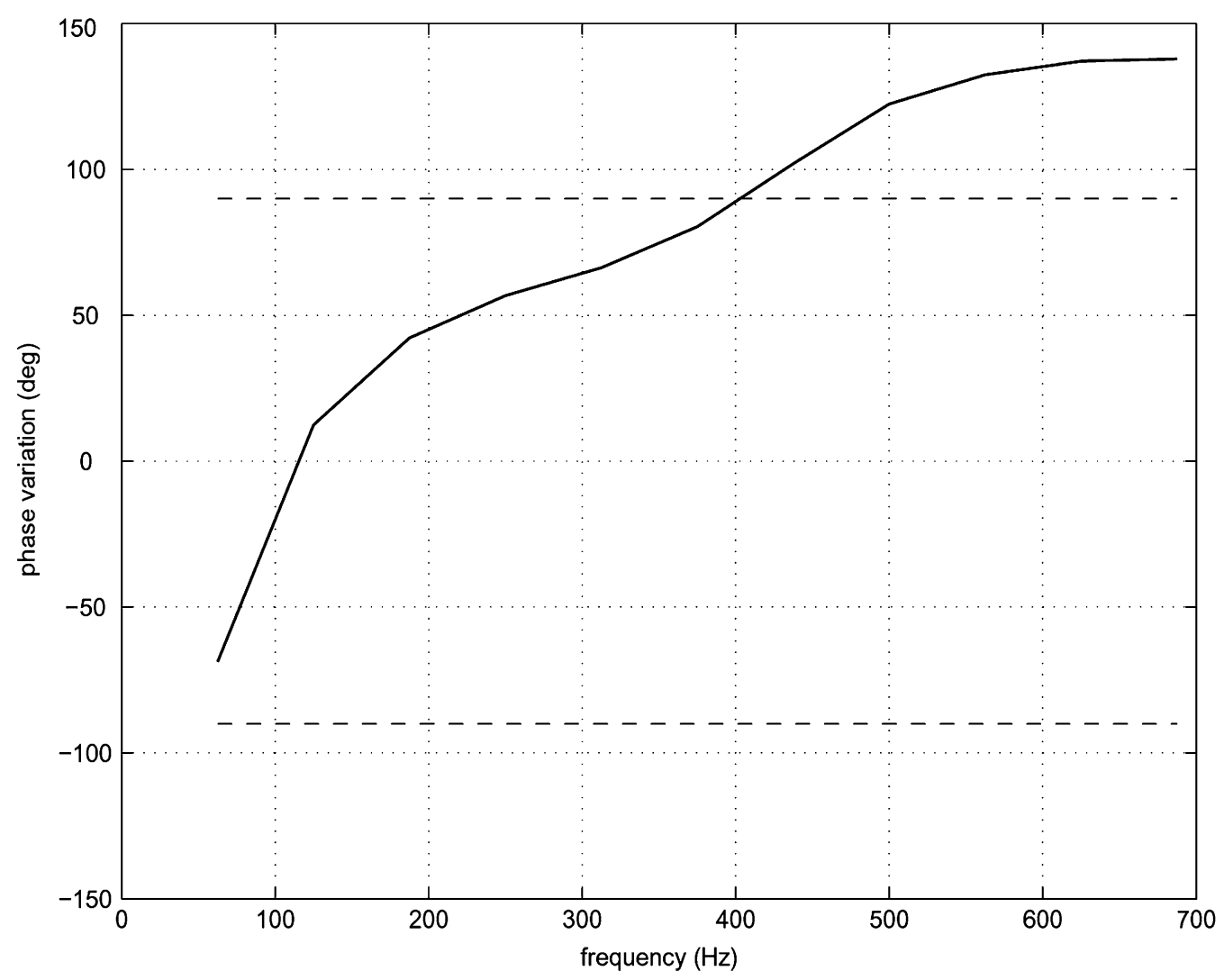

Fig. 4. Phase difference between the position 1 and position 2, which approximates the phase estimation error when the ear-cup is moved from the position 1 to position 2.

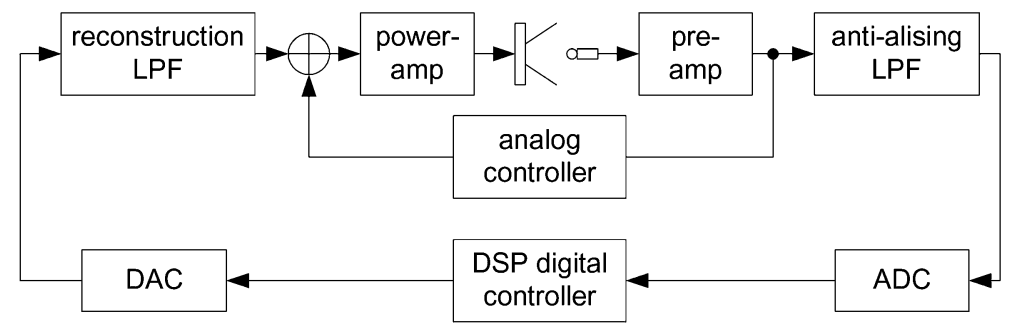

Fig. 5. Hybrid analog/digital ANC system.

\section{HyBRID ANC HEADSET SYSTEM}

In this section, we will analytically show that adding an analog feedback loop into the controller can effectively reduce the phase fluctuation of the secondary path and describe how to design the analog filter.

\section{A. Plant Fluctuation Reduction}

Adding an analog feedback loop to the digital ANC headset results in a hybrid analog/digital ANC system, as shown in Fig. 5. The block diagram of the hybrid ANC system using the FXLMS algorithm is illustrated in Fig. 6. It shows that the transfer function of the overall secondary path with the analog feedback loop becomes

$$
G^{\prime}(s)=\frac{G(s)}{1+G(s) H(s)}
$$

where $H(s)$ and $G(s)$ are the transfer functions of the analog controller and the secondary path respectively, and the Laplace transform rather than the $z$-transform is used to avoid confusing since the added feedback system is analog. The relation between the Laplace transform for the secondary path and its equivalent $z$-transform counterpart can be easily obtained once the sampling rate is given [2].

The accuracy of the overall secondary path estimate $\hat{G}^{\prime}(s)$ determines the stability of the FXLMS algorithm in the hybrid ANC system. Writing $G(s)$ and $H(s)$ in polar forms as $G(s)=$ $|G| e^{j \theta_{G}}$ and $H(s)=|H| e^{j \theta_{H}}$, and substituting them into (2) gives

$$
G^{\prime}(s)=\frac{|G| e^{j \theta_{G}}}{1+|G H| e^{j\left(\theta_{H}+\theta_{G}\right)}}=\frac{|G| e^{j \theta_{G}}}{|A| e^{j\left(\theta_{H}+\theta_{G}+\varphi\right)}}
$$

where

$$
|A|=\sqrt{1+2|G H| \cos \gamma+|G H|^{2}}
$$

$\gamma=\theta_{H}+\theta_{G}$, and $\varphi$ is determined by

$$
|A| \cos (\gamma+\varphi)=1+|G H| \cos \gamma
$$


or

$$
|A| \sin (\gamma+\varphi)=|G H| \sin \gamma
$$

Multiplying both sides of (5) by $\cos \gamma$ and (6) by $\sin \gamma$, and summing the results, we obtain

$$
\cos \varphi=\frac{1}{|A|}(\cos \gamma+|G H|) .
$$

Similarly, multiplying (5) by $\sin \gamma$ and the result is subtracted by the result of multiplying (6) with $\cos \gamma$, we have

$$
\sin \varphi=\frac{-1}{|A|} \sin \gamma
$$

For comparison purpose, we investigate the phase fluctuation of $G^{\prime}(s)$ under the same situations as those given in Section II. The phase variation of $G^{\prime}(s)$ from position 1 to position 2 determines the stability of the FXLMS algorithm.

From (3), the phase of $G^{\prime}(s)$ can be obtained as $\theta_{G^{\prime}}=-\left(\theta_{H}+\varphi\right)$, and the phase change from position 1 to position 2 is given by

$$
\left|\theta_{12}^{\prime}\right|=\left|\theta_{G_{1}^{\prime}}-\theta_{G_{2}^{\prime}}\right|=\left|\varphi_{1}-\varphi_{2}\right|
$$

where the subscripts 1 and 2 denote the parameters corresponding to positions 1 and 2 , respectively. Therefore, we obtain

$$
\cos \theta_{12}^{\prime}=\cos \varphi_{1} \cos \varphi_{2}+\sin \varphi_{1} \sin \varphi_{2}
$$

Substituting (7) and (8) into (10) with some manipulations gives

$$
\begin{aligned}
\cos \theta_{12}^{\prime}=\frac{1}{\left|A_{1} A_{2}\right|}[\cos ( & \left.\theta_{G_{1}}-\theta_{G_{2}}\right)+\left|G_{1} H\right| \cos \gamma_{2} \\
& \left.+\left|G_{2} H\right| \cos \gamma_{1}+\left|G_{1} G_{2} H^{2}\right|\right]
\end{aligned}
$$

For the stability of the FXLMS algorithm, we should ensure that $\left|\theta_{12}^{\prime}\right|<90^{\circ}$, or

$$
\cos \theta_{12}^{\prime}>0
$$

Let $\theta_{12}=\theta_{G_{1}}-\theta_{G_{2}}$ be the phase change of the secondary path if there is no analog controller. We can express (12) as

$$
\cos \theta_{12}+K>0
$$

where $K=\left|G_{1} H\right| \cos \gamma_{2}+\left|G_{2} H\right| \cos \gamma_{1}+\left|G_{1} G_{2} H^{2}\right|$. We note that if there is no analog feedback loop, the constraint on the phase variation is $\cos \theta_{12}>0$. Thus if $K>0$ in (13), the phase variation constraint on the original secondary path $G(z)$ is relaxed. That is, the phase fluctuation for the overall secondary path $G^{\prime}(z)$ is reduced.

Assuming that $\theta_{G_{1}}$ and $\theta_{G_{2}}$ are known, we can always have $K>0$ with an appropriate design of the analog controller. Because the maximum absolute phase difference between two signals is $180^{\circ}$, we have $\left|\theta_{G_{1}}-\theta_{G_{2}}\right| \leq 180^{\circ}$. In addition, because $\gamma_{1}=\theta_{H}+\theta_{G_{1}}$ and $\gamma_{2}=\theta_{H}+\theta_{G_{2}}$, the phase of the analog controller $\theta_{H}$ can be regarded as a phase shifter that operates on both $\theta_{G_{1}}$ and $\theta_{G_{2}}$. By choosing $\theta_{H}$ properly, we can simultane- ously shift $\gamma_{1}$ and $\gamma_{2}$ within the range of $\left(-90^{\circ}, 90^{\circ}\right)$. We then have $\cos \gamma_{1}>0$ and $\cos \gamma_{2}>0$, and obtain $K>0$ in (13).

\section{B. Discussion}

The above analysis can be extended to more general cases where the ear-cup is adjusted at different positions. In such scenarios, we have $\gamma_{i}=\theta_{H}+\theta_{G_{i}}$, where $i=1, \ldots, M$ for $\mathrm{M}$ possible ear-cup positions, and $\theta_{G_{i}}$ is the phase of the secondary path at position i. Following the similar derivation given in the previous example, we can conclude that if $\cos \gamma_{i}>0$, or equivalently $\left|\gamma_{i}\right|<90^{\circ}$ for all $\mathrm{i}$, the stability of the FXLMS algorithm at all positions can be improved. Without losing generality, we still assume that position 1 is the normal wearing position where the ear-cup covers the ear properly, and position 2 corresponds to the worst case where the ear-cup is totally pulled away from the ear. In this case, we have $\left|\theta_{12}\right|>\left|\theta_{1 i}\right|, i \neq 2$, where $\theta_{1 i}=\theta_{G_{1}}-\theta_{G_{i}}$ is the phase variation of the secondary path when the position is changed from position 1 to position $\mathrm{i}$. If $\theta_{H}$ can shift both $\gamma_{1}$ and $\gamma_{2}$ within the range of $\left(-90^{\circ}, 90^{\circ}\right)$, it can also work for all other $\gamma_{i}$ since $\left|\theta_{1 i}\right|=\left|\theta_{G_{1}}-\theta_{G_{i}}\right| \leq 180^{\circ}$ for all $\mathrm{i}$. We then have positive $\mathrm{K}$ for all ear-cup positions. This observation implies an important fact for practical implementation that the adjustment of $\mathrm{K}$ needs only be made based on a worst-case scenario.

In real applications, it is difficult to determine which position corresponds to the "worst-case" scenario. In real-time experiments, we always observe that the farther the ear-cup is pulled away from the ear, the lager the secondary path variation tends to appear. On another front, since a practical headset consists of a pair of ear-cups connected by an elastic belt, the ear-cups can only be pulled away from the ears to some extend. Thus we can consider the ear-cup at the farthest position from the ear as the "position 2," which may not correspond to the true worst-case. Another problem is that different users may have different worst-case scenario. The difference, however, is usually small since the ear-cups are normally designed to be able to cover the ears well for most individuals. In general, the system is always designed to have "stability margin."

Finally we note that, to be more strictness, the stability condition for the feedback ANC consists of two terms [10], [11], where the first term corresponds to the $90^{\circ}$ condition and second term is usually a small value which is determined by the spectral properties of $G(z)$ and the noise. Therefore, at least increase of the first term may enhance the stability.

\section{Analog Controller Design}

Equation (13) suggests a method for designing the analog controller to improve the robustness of the FXLMS algorithm. However, it does not consider the stability and other characteristics of the analog feedback loop, which are also essential to the overall ANC system. For example, we observe from (13) that the value of $K$ is determined by the analog loop gain. Normally the larger the analog loop gain, the larger the positive $K$ that can be used. Thus the FXLMS algorithm requires the analog loop gain to be as large as possible for its robustness. However, the large analog loop gain may drive the analog feedback loop itself unstable, although it may be desired for the noise attenuation purpose. Therefore, it is not always possible, 
or necessary, to have $K>0$ for all frequencies by considering the stability and other factors of the analog feedback loop. The adjustment of $K$ should particularly focus on those frequencies with large phase fluctuations and high level of noises. Although many methods for designing the analog controller can be found in literatures [13]-[16], they are all developed for analog ANC systems. Thus they must be modified for the hybrid analog/digital ANC systems in order to improve the stability of the FXLMS algorithm.

In this paper, we modified the technique presented in [13] for designing the analog controller of the hybrid headsets. The design of the robust analog controller can be achieved by maximizing the noise attenuation subject to four constraints including controller stability, prescribed stability margin, waterbed effect, and high-frequency uncertainty stability. Considering the stability of the FXLMS algorithm, we add (13) as the fifth constraint to the optimization given in [13]. The value of $K$ is determined according to the stability margin that is required by the FXLMS algorithm. For example, if the headset is used for a particular situation where the frequency range of the high level noise is known, $K$ may be set to a large value for those frequencies.

Note that (13) may not be easy to satisfy for some applications. As an alternative, we may set the fifth constraint as

$$
\left|\theta_{H}+\theta_{G_{i}}\right|<90^{\circ}, i=1,2
$$

Initially, this constraint should be only on frequencies with large secondary path phase fluctuations. If the optimization results in $\cos \theta+K<0$ for some frequencies, we should include more frequencies on the constraint of (14), and redo the optimization until (13) is satisfied for all frequencies. In practice, we may use either (13) or (14) as the fifth constraint.

Another factor that should be highlighted is that when implementing the constraint of (13) or (14), it is only necessary to estimate the frequency responses of the secondary path when the ear-cup covers the ear properly and the ear-cup is at the farthest position away from the ear, which corresponds to the worst case of the phase fluctuations.

\section{REAL-TIME IMPLEMENTATION AND RESULTS}

In this section, we implement a hybrid analog/digital ANC headset with the feedback FXLMS algorithm as illustrated in Fig. 6 for real-time experiments.

\section{A. Experiment Setup}

An experimental setup is built to measure the performance of hybrid digital/analog ANC headset. A loudspeaker is used to play noise to simulate a noisy environment. The original noise is a tone embedded in wideband noise. Other equipment includes a dummy head with an ANC headset and data acquisition tools. The signal is captured in the first stage of pre-amplifier of the error microphone. This signal is digitized by the sound card inside the computer through its line-in port. The sampling rate of sound card is set at $8 \mathrm{kHz}$. When the data is captured, the noise level near the ear-cup is about $80 \mathrm{~dB}$ measured by a sound level meter.

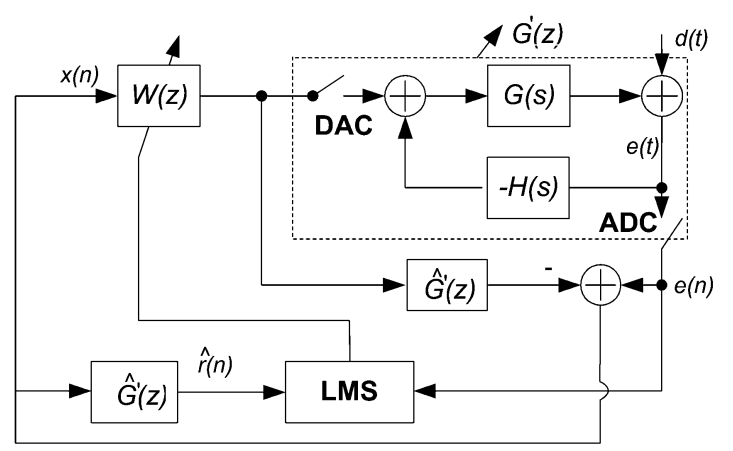

Fig. 6. Hybrid analog/digital ANC headset with the feedback FXLMS algorithm.

The analog controller is designed so that it can be put into or taken out from the system in order to compare ANC performance. Specifically, it is a second-order highpass filter with transfer function expressed as

$$
H(s)=\frac{0.0307 s^{2}+8.5323 s+10511}{0.018 s^{2}+4.78 s+11213} .
$$

The Bode plots of the analog controller $G(s)$ and the feedback loop $G(s) H(s)$ are shown in Figs. 7 and 8, respectively.

The digital controller, implemented on the ADSP21065 digital signal processor (DSP), is a 64-tap adaptive FIR filter with the leaky normalized FXLMS algorithm. The microphone, pre-amplifier, power amplifier, and loudspeaker are shared by the analog and digital controllers that are connected by the analog-to-digital converter (ADC) and the digital-to-analog converter (DAC). The sampling rate is $2 \mathrm{kHz}$, and the cutoff frequency of the anti-aliasing and reconstruction lowpass filters is $600 \mathrm{~Hz}$. The working frequency of this prototype is from $100 \mathrm{~Hz}$ to $600 \mathrm{~Hz}$, and the headset shells that cover the ears can effectively attenuate noise higher than $600 \mathrm{~Hz}$.

\section{B. Experiment Results}

Fig. 9 compares the noise cancellation performance of the digital, analog and hybrid ANC headsets by showing their output signals (after the pre-amplifier) of the error microphones over $2500 \mathrm{~ms}$ when the ear-cups are well covered around the ears. The vertical dash lines in Fig. 9 indicate the time instant that the ANC operations are switched on. It is clearly shown that all of the ANC systems can suppress the noise to some extend; the hybrid ANC system has the best performance among the three.

Fig. 10 plots the spectra of the error signals corresponding to Fig. 9 after the ANC operations are switched on. The curves are obtained by calculating 512 points FFT with $50 \%$ overlap rectangular window. The spectrum of the original noise (a 230 $\mathrm{Hz}$ tone embedded in wideband noise) is also plotted in Fig. 10. We can clearly observe that the digital ANC headset can effectively reduce the tonal noise but not the wideband noise, while the analog ANC headset has problem in suppressing tonal noise though it can attenuate wideband noise. The hybrid ANC headset can effectively attenuate both narrowband and wideband noises, and thus has the best performance among the three. 

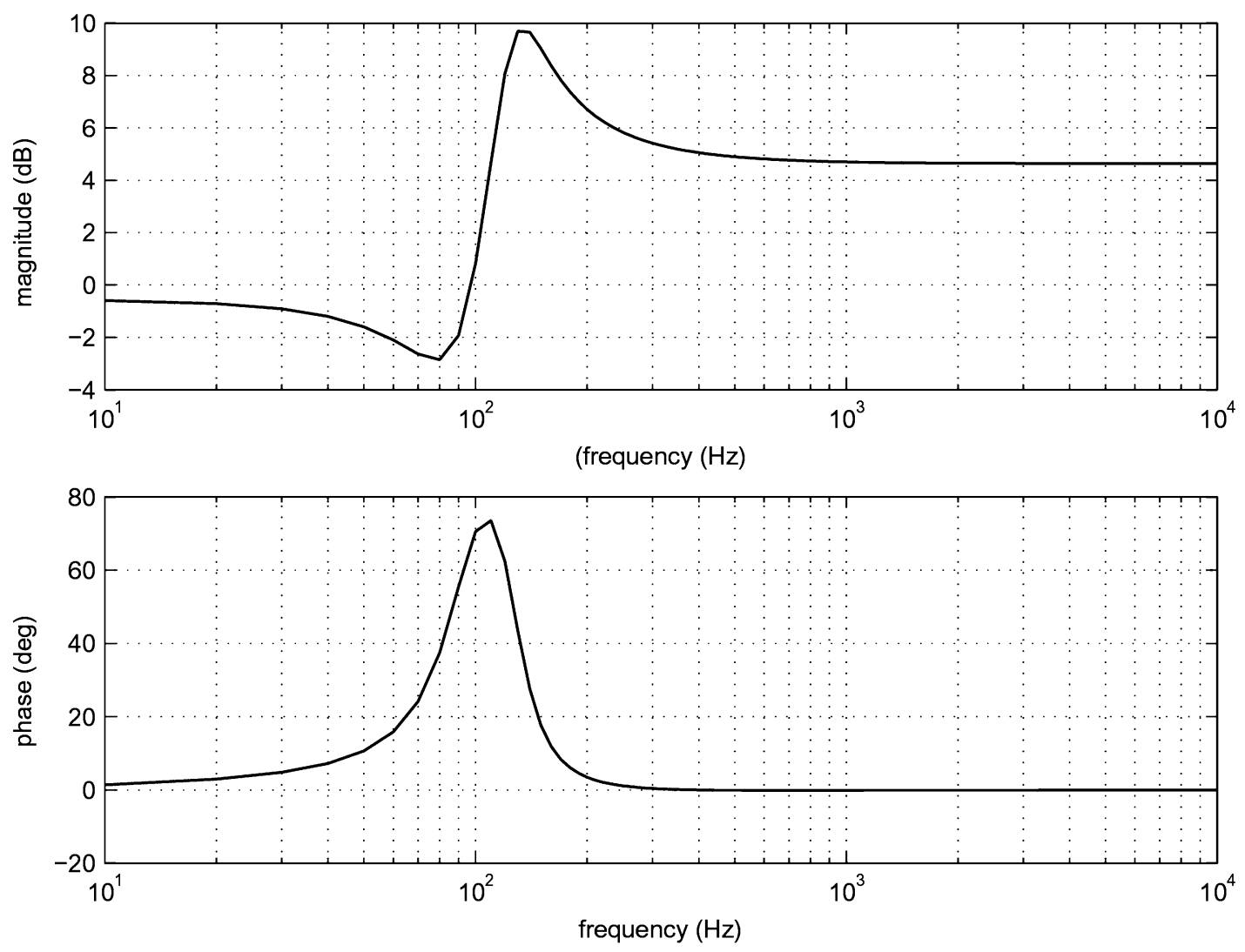

Fig. 7. Bode plot of the open-loop gain of the analog feedback controller $G(s)$.
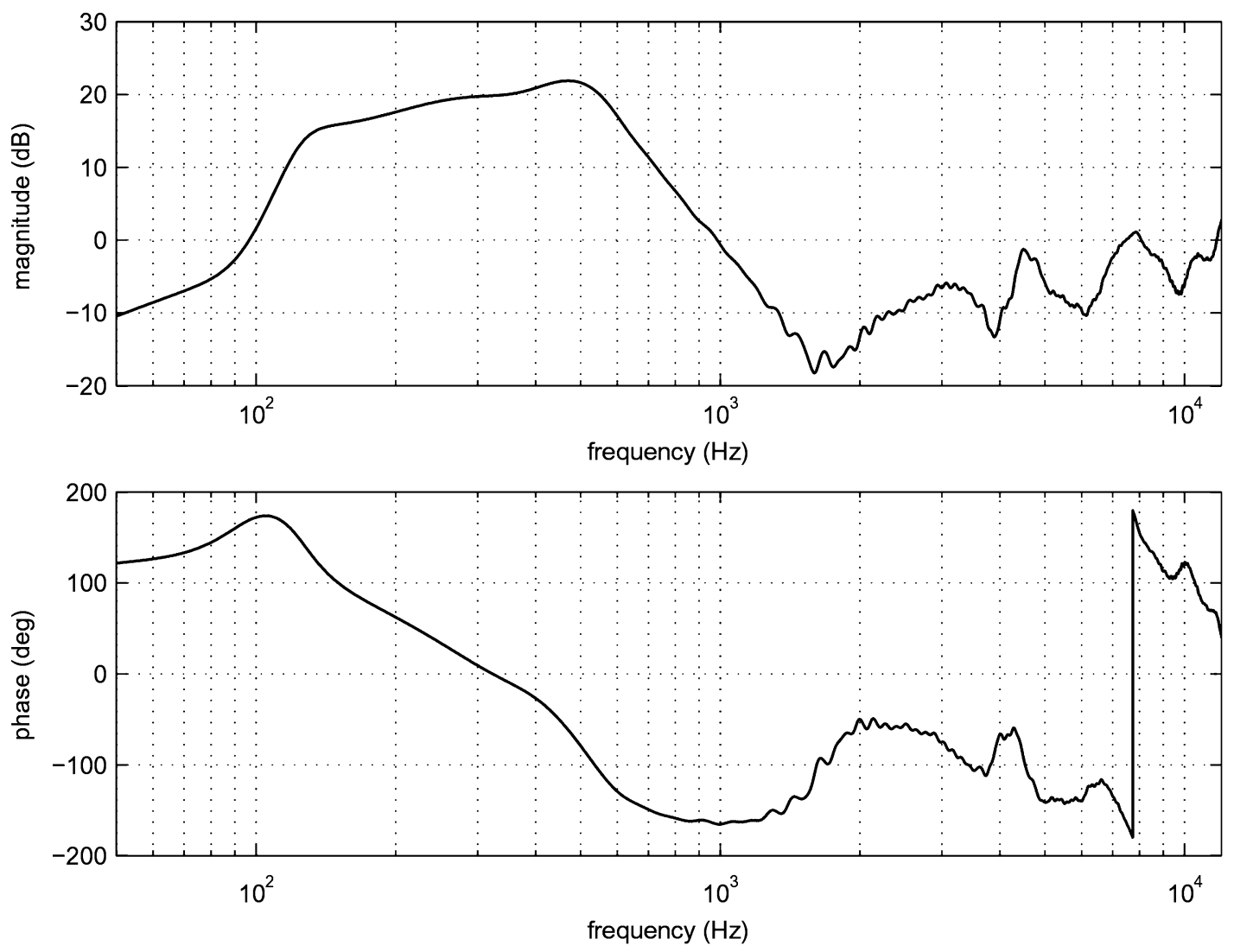

Fig. 8. Bode plot of the open-loop gain of the analog feedback system $G(s) H(s)$. 

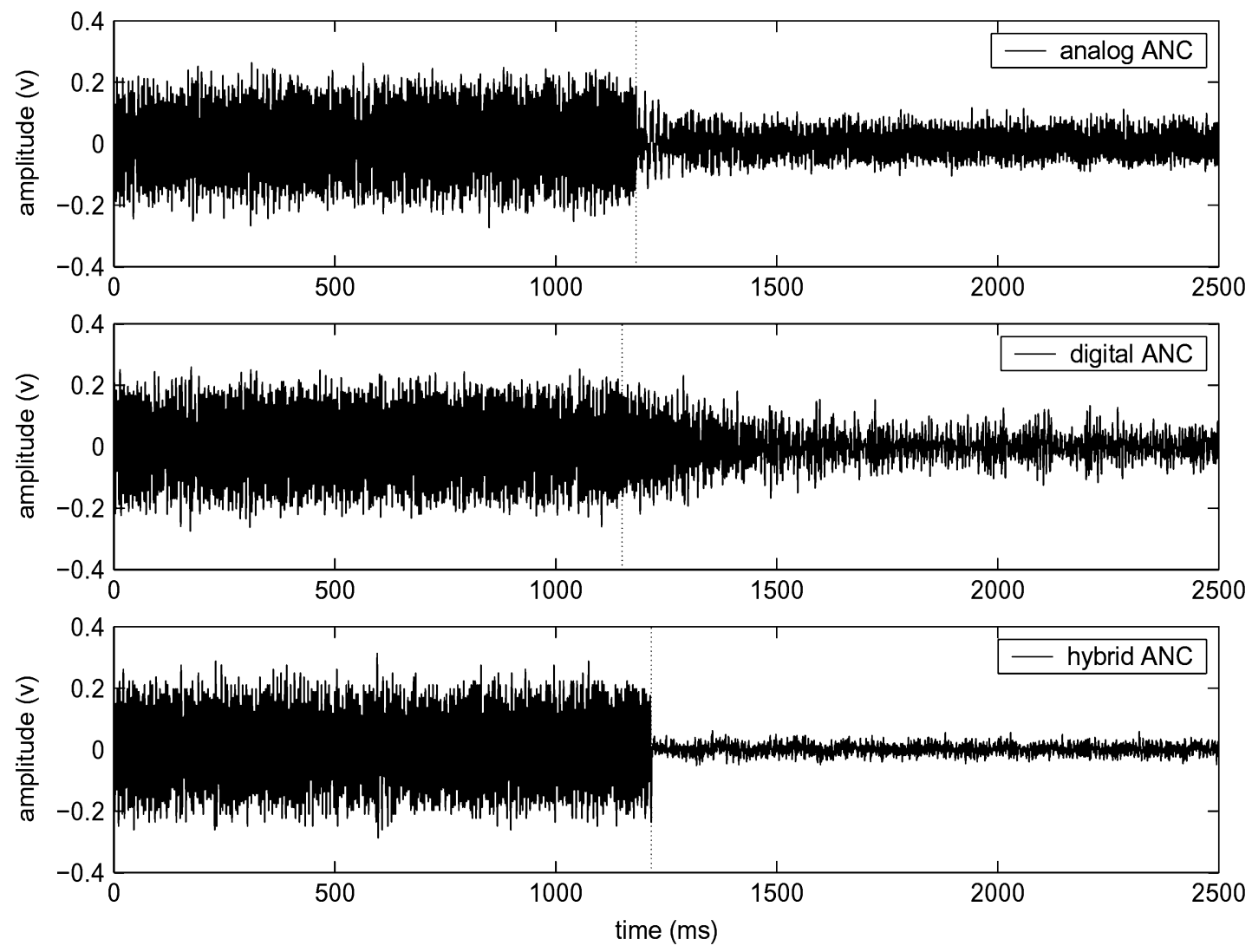

Fig. 9. Error signal amplitude (in unit volts) after the pre-amplifier for the analog, digital and hybrid ANC headsets respectively.

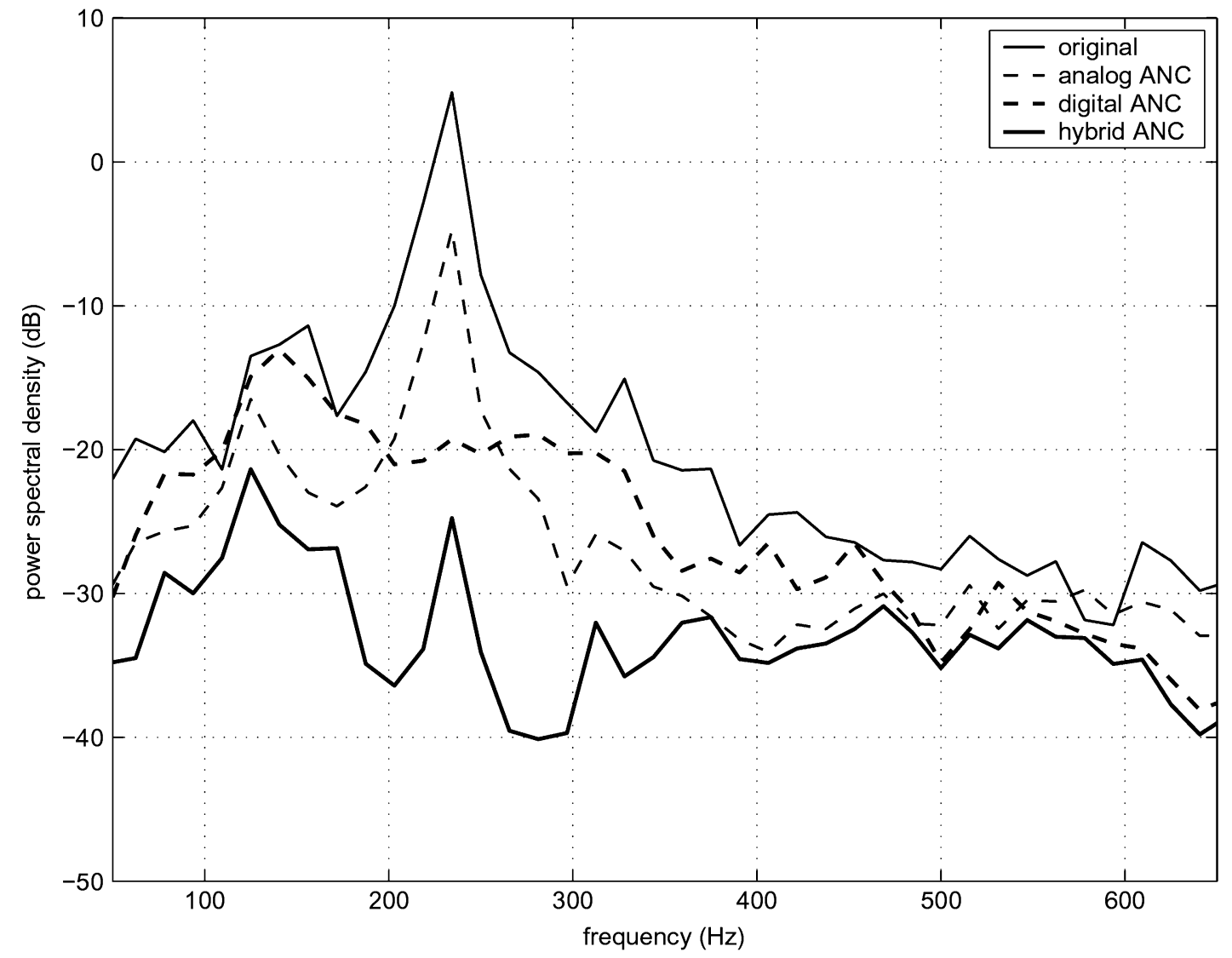

Fig. 10. Spectra of the error signals for analog, digital and hybrid ANC headset systems. 


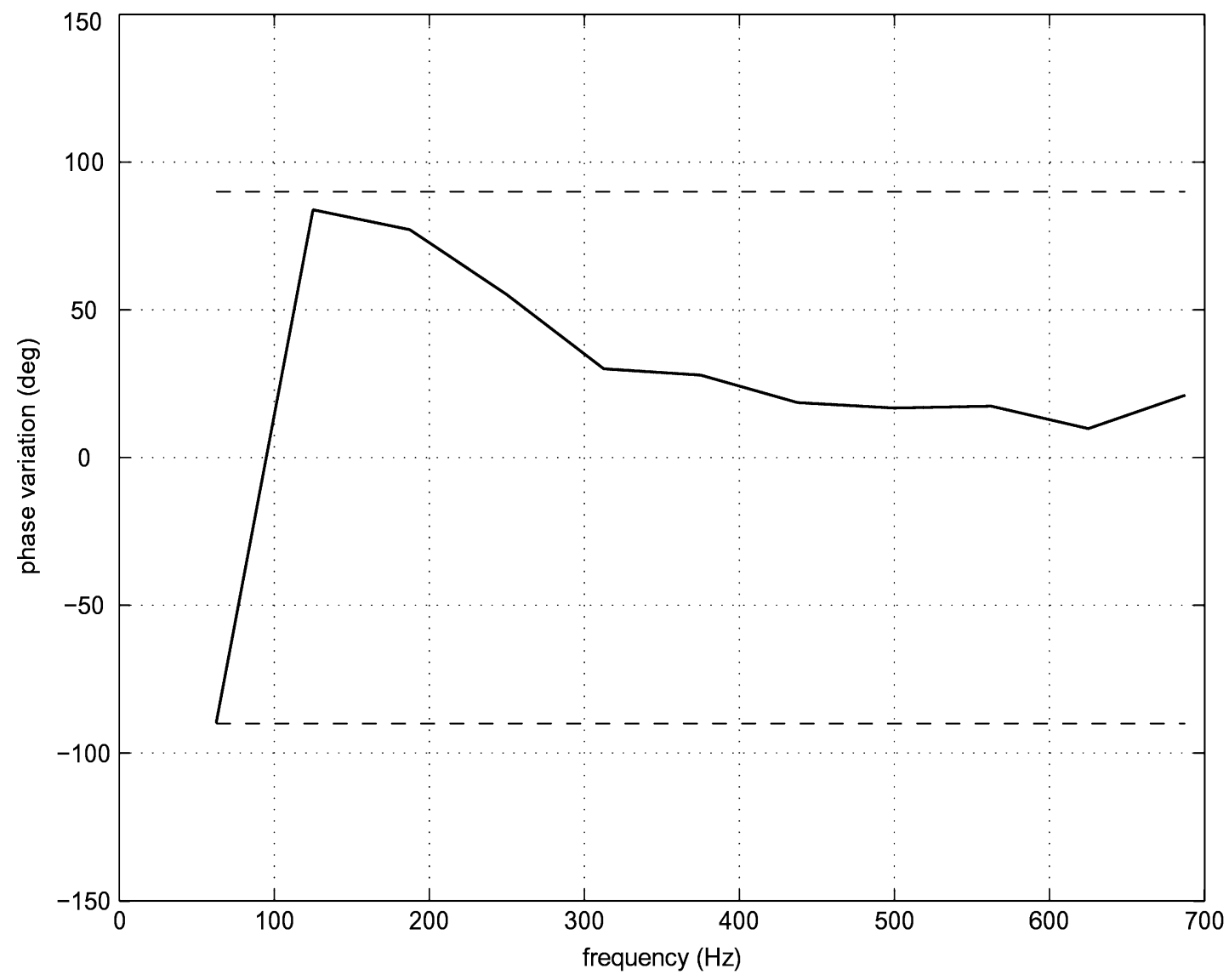

Fig. 11. Measured phase error with analog feedback loop.

We only use the hybrid ANC headset system for the following experiments. First, the phase variation of the overall secondary path from the position 1 to position 2 with the analog controller is plotted in Fig. 11. By comparing Fig. 11 with Fig. 4 (without analog feedback loop), we can show that the analog controller effectively reduces the phase fluctuation as expected. We also observe that ANC with analog feedback loop has larger phase fluctuation at some frequencies below $250 \mathrm{~Hz}$, which implies negative $K$ in (13) at those frequencies. However, the phase fluctuations in the working frequency range are all within $90^{\circ}$. This indicates that it is not necessary (even if possible) to have $K>0$ for all frequencies.

Fig. 12 shows the output signal (after the pre-amplifier) of the error microphone over $16000 \mathrm{~ms}$. The plot is divided into seven time slots. Time slot 1 shows the original noise (a $350 \mathrm{~Hz}$ tone embedded in a white noise) captured by the microphone when the ANC is turned off and the ear-cup covers the ear properly. Time slot 2 displays the results when only the analog controller is switched on, and it shows that the analog controller can reduce broadband noise. Time slot 3 shows that both the analog and digital controllers are working, and the noise is obviously further reduced in comparison with the time slot 2 . From time slot 4 , the ear-cup is being pulled away from the ear until at time slot 5 the ear-cup is at its farthest position, i.e., $4.5 \mathrm{~cm}$ away from the ear. Then during time slot 6 , the ear-cup is gradually being put back to cover the ear, and finally at time slot 7 it resumes the normal wearing position. In this experiment, we assume the position corresponding to time slot 5 as the "worst case", i.e., position 2. It is clearly shown in Fig. 12 that though the noise level increases sharply during time slots 4, 5, and 6, the system is still stable. At time slot 7, the feedback FXLMS algorithm is re-converged, and thus the noise is reduced back to a level similar to that in time slot 3 . The time slots 4 and 6 correspond to different ear-cup positions other than "position 2." The stability of the system during time slots 4 and 6 verified our previous statement that the analog controller needs only be designed based on the worst-case scenario. In fact, we have tested our hybrid ANC headset for many ear-cup positions and observed that the headset system was always stable. For example, we examined the case that the headset was taken away from the dummy head and put on the table. We found that, even doing so, the system is still stable and the error microphone is similar to Fig. 12.

In Fig. 13, we plot the spectra of signals over the working frequency range (below $650 \mathrm{~Hz}$ ) for time slots 1,2 , and 3 as shown in Fig. 12 to demonstrate the noise cancellation performance of the hybrid ANC headset. Like those shown in Fig. 10, the spectra are calculated using 512 points FFT with $50 \%$ overlap rectangular window. The original noise in time slot 1 consists of $350 \mathrm{~Hz}$ tonal noise mixed with broadband white noise. After the analog controller is switched on, the broadband noise in time slot 2 is reduced. It shows that the analog controller is able to attenuate broadband noise, but failed to control narrowband noise. When the digital controller is turned on (time slot 3 ), the tonal 


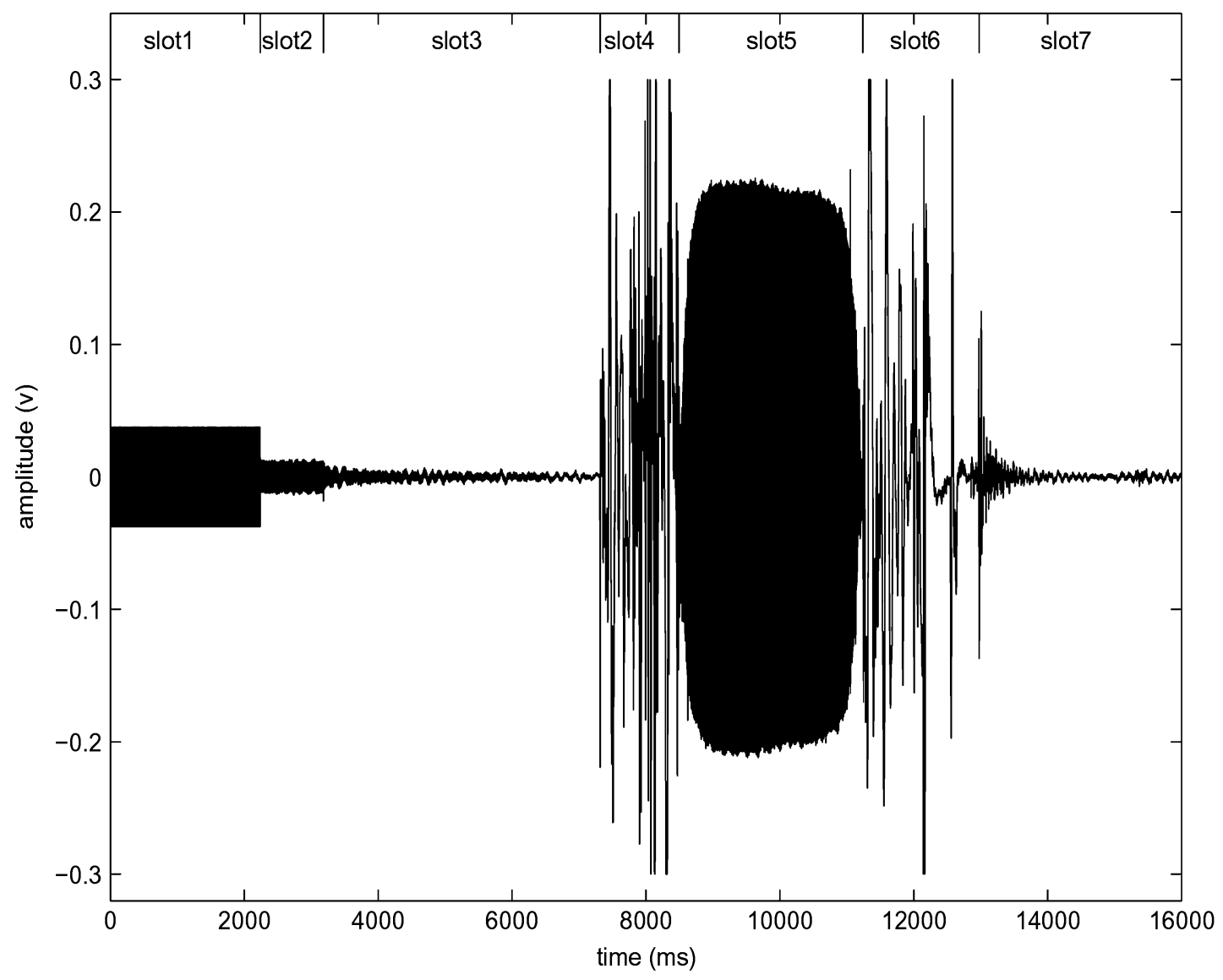

Fig. 12. Error signal amplitude (in unit volts) after the pre-amplifier.

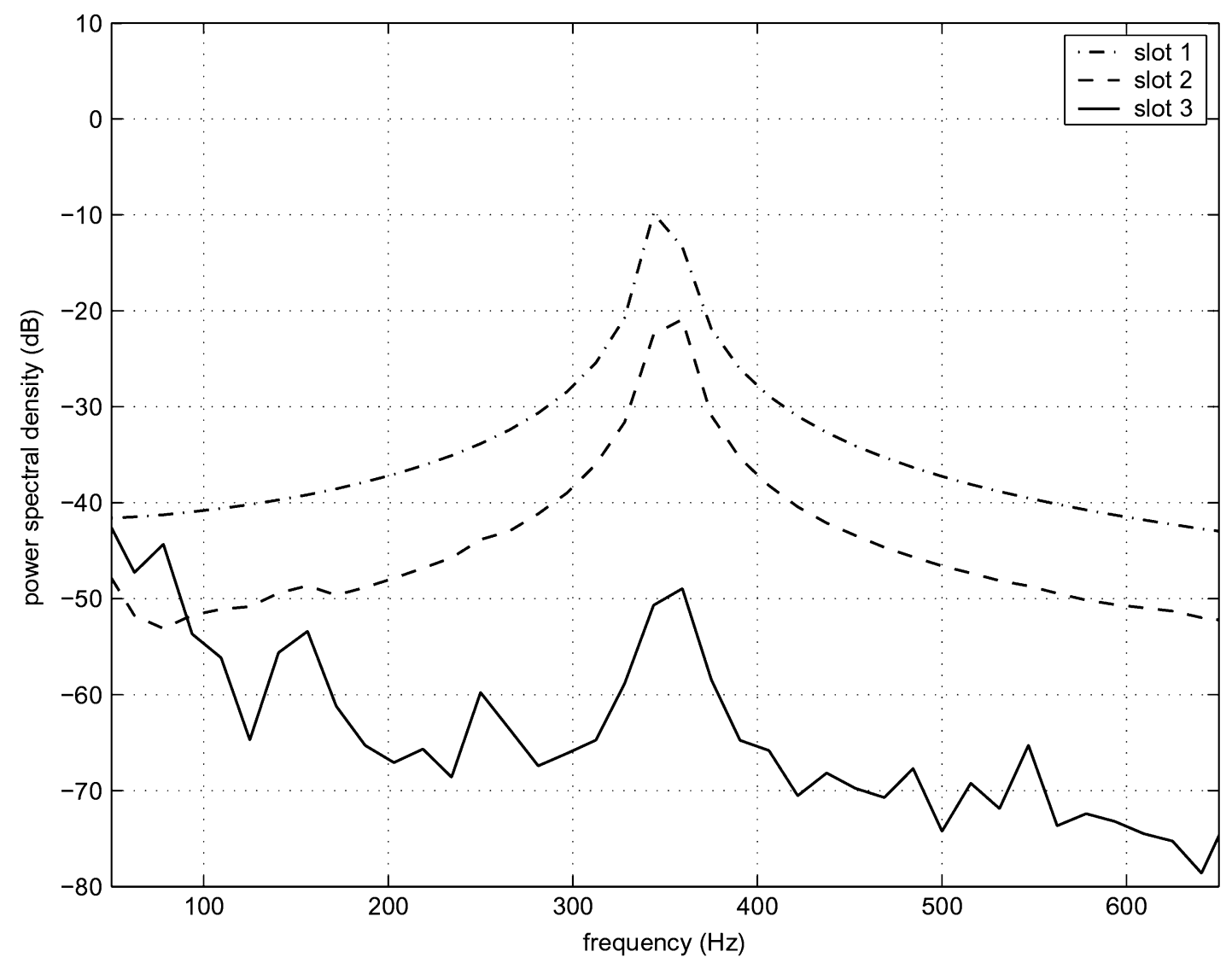

Fig. 13. Spectra of signals in time slot 1 , time slot 2 , and time slot 3 . 


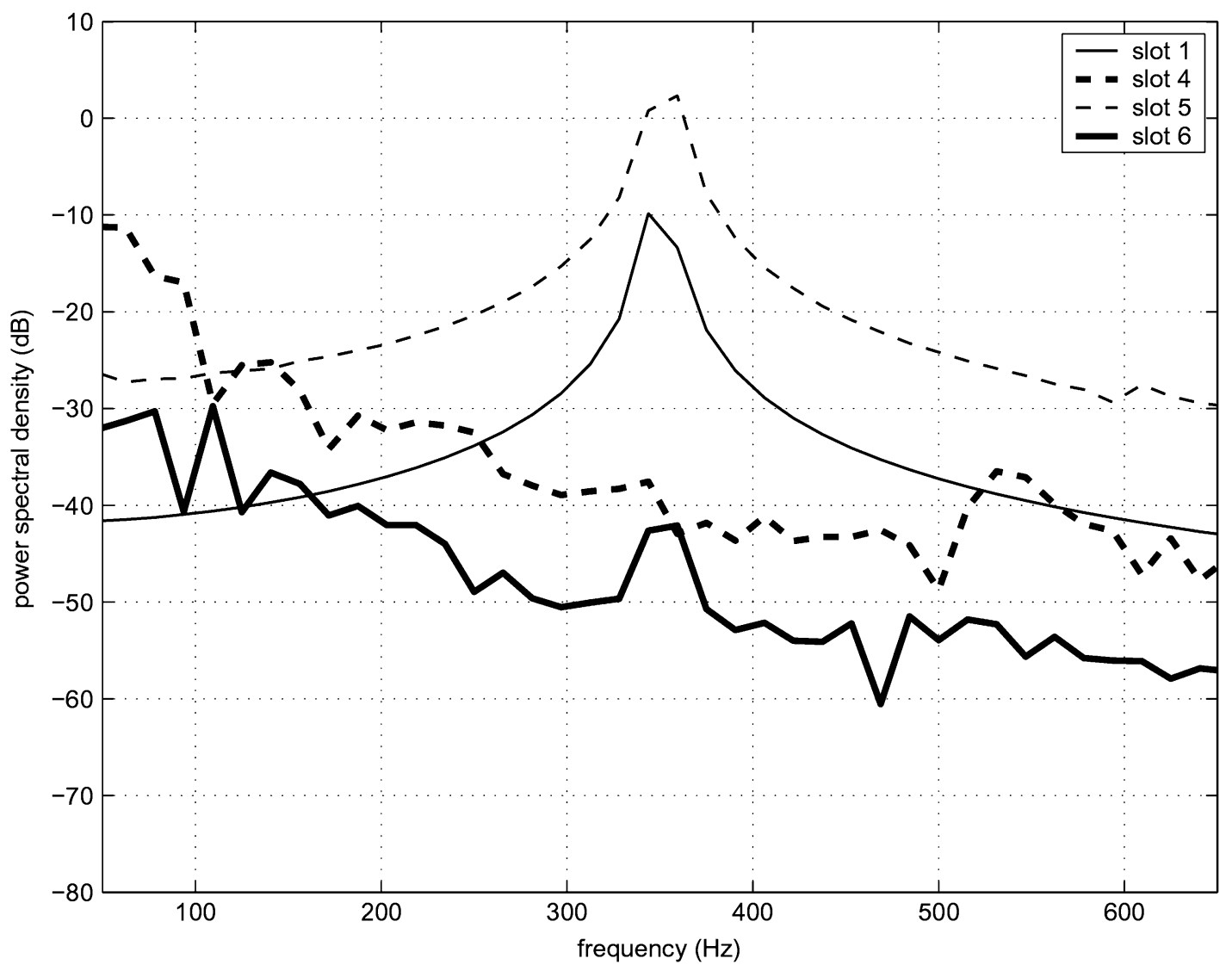

Fig. 14. Spectra of signals in time slot 1 , time slot 4 , time slot 5 , and time slot 6 .

noise is reduced. This shows the digital controller is effective in canceling narrowband noise, thus compensates for the deficiency of the analog controller.

In Fig. 14, we plot the spectra of signals for time slots 1, 4, 5, and 6 shown in Fig. 12, respectively, to have a better view of the stability of the hybrid ANC headset. Considering that the signals may have different spectra at different time instants during either time slot 4 or 6 , we only plot one typical spectrum for each of time slots 4 and 6 , corresponding to the time instants of $7990 \mathrm{~ms}$ and $12960 \mathrm{~ms}$, respectively. Fig. 14 clearly shows that the system remains stable during time slots 4,5 , and 6 , and time slot 5 corresponds to the worst-case scenario. It is also interesting to observe that the tonal noise is still suppressed to some extend for both of the specified time instants in time slots 4 and 6 , implying that the FXLMS algorithm is not totally ineffective at those points.

The plots corresponding to time slots 4,5 , and 6 will be totally black if there is no analog feedback loop, which indicates that the ANC system becomes unstable when the ear-cup is pulled away from the ear. It also shows that the stability cannot be resumed after the ear-cup is put back to cover the ear. The experiment results verify that the proposed hybrid analog/digital ANC headset can effectively reduce plant fluctuation and result in a more robust system. In addition, the hybrid ANC systems can cancel both narrowband and broadband noises, resulting in a more satisfactory performance.

\section{CONCLUSION}

This paper investigated the robust hybrid analog/digital ANC headset system using the feedback FXLMS algorithm. We showed the analog feedback loop can effectively reduce the plant fluctuations and results in a more robust ANC system. The design of the analog controller was also highlighted. Finally, a practical hybrid feedback ANC headset was built and used for real-time experiments to verify the analysis results.

\section{REFERENCES}

[1] S. M. Kuo and D. R. Morgan, Active Noise Control Systems: Algorithms and DSP Implementations. New York: Wiley, 1996.

[2] S. J. Elliott, Signal Processing for Active Noise Control. London, U.K.: Academic, 2001.

[3] H. F. Olson and E. G. May, "Electronic sound absorber," J. Acoust. Soc. Amer., vol. 25, pp. 1130-1136, 1953.

[4] M. E. Hawley, "Acoustical interference for noise control," Noise Contr., vol. 2, pp. 61-63, 1956

[5] W. F. Meeker, "Components characteristics for an active ear defender," J. Acoust. Soc. Amer., vol. 29, pp. 1252-1252, 1957.

[6] B. Rafaely, "Active noise reducing headset," in Proc. Online Symp. Electronics Engineers, 2002.

[7] D. R. Morgan, "An analysis of multiple correlation cancellation loops with a filter in the auxiliary path," IEEE Trans. Acoust., Speech, Signal Process., vol. ASSP-28, pp. 454-467, Aug. 1980.

[8] A. K. Wang and W. Ren, "Convergence analysis of the multi-variable filtered-x LMS algorithm with application to active noise control," IEEE Trans. Signal Process., vol. 47, pp. 1166-1169, 1999.

[9] S. D. Snyder and C. H. Hansen, "The effect of transfer function estimation errors on the filtered-X LMS algorithm," IEEE Trans. Signal Process., vol. 42, pp. 950-953, 1994. 
[10] H. Sakai and S. Miyagi, "Analysis of the adaptive algorithm for feedback-type active noise control," Signal Process., vol. 83, pp. 1291-1298, 2003.

[11] — , "Analysis of the adaptive algorithm for feedback-type active noise control," in Proc. IEEE Int. Conf. Acoustics, Speech, Signal Processing, 2001, pp. 3841-3844

[12] W. S. Gan and S. M. Kuo, "An integrated audio and communication active noise control headsets," IEEE Trans. Consumer Electron., vol. 48, pp. 242-247, May 2002.

[13] S. H. Yu and J. S. Hu, "Controller design for active noise cancellation headphones using experimental raw data," IEEE/ASME Trans. Mechatronics, vol. 6, pp. 483-490, Dec. 2001.

[14] M. S. Bai and D. Lee, "Implementation of an active headsets by using $H_{\infty}$ robust control theory," J. Acoust. Soc. Amer, vol. 102, pp. 2184-2190, 1997

[15] R. R. Leitch and M. O. Tokhi, "Active noise control systems," in Proc. Inst. Elect. Eng. A, vol. 134, 1987, pp. 525-546.

[16] P. D. Wheeler, "Voice Communication in the Cockpit Noise Environment-The Role of Active Noise Reduction," Ph.D. dissertation, Univ. Southampton, Southampton, U.K., 1986.

[17] S. J. Elliott and P. A. Nelson, "Algorithms for the active control of periodic sound and vibration," in ISVR Tech. Memo.. Southampton, U.K., 1986.

[18] H. Lan, M. Zhang, and W. Ser, "A weight-constrained FXLMS algorithm for feed-forward active noise control systems," IEEE Signal Process. Lett., vol. 9, no. 1, pp. 1-4, Jan. 2002

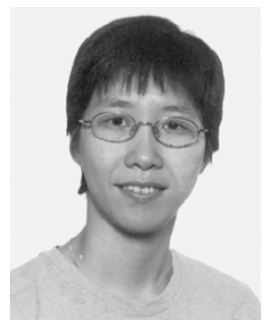

Ying Song received the B.S. degree from Southeast University, Nanjing, China (formerly Nanjing Institute of Technology) and the M.S. degree from the Secondary Academic of Astronautics Ministry, Beijing, China, in 1985 and 1988, respectively, both in electrical engineering.

From 1988 to 1993, she was with Beijing Institute of Radio Measurement, specializing in the realtime application, firmware design and development of signal processing system used in radar and communications. From 1994 to 1998, she was with DSO National Laboratory, Singapore, where she was involved in the analysis and development of signal processing techniques associated with image processing, adaptive system, signal detection and estimation. Since 1998 she has been with Centre for Signal Processing, Singapore, which was merged into Institute for Infocomm Research, Singapore in 2002, where she has been working on acoustic and audio signal processing, communications, array processing, medical signal processing, and active noise control.

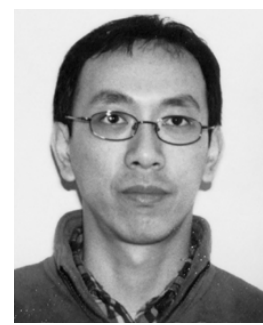

Yu Gong received the B.S. and M.S. degree from University of Electronic Science and Technology of China in 1992 and 1995, respectively, and the Ph.D. degree from the National University of Singapore in 2002 , all in electrical engineering.

In 2000, he took a position as a Research Engineer in the Centre for Signal Processing (CSP), Singapore, and was later promoted to a senior position when CSP was merged into Institute for Infocomm Research in 2002. Since 2003, he has been a Research Fellow in Queen's University of Belfast, Belfast, U.K. His research interests are adaptive filters, signal processing, and digital communications.

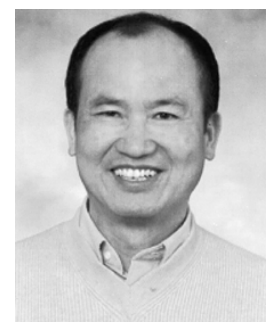

Sen M. Kuo (M'84-SM'04) received the B.S. degree from National Taiwan Normal University in 1976 and the M.S. and Ph.D. degrees from the University of New Mexico, Albuquerque, in 1983 and 1985, respectively.

He is a Professor and Chair at the Department of Electrical Engineering, Northern Illinois University, Dekalb. In 1993, he was with Texas Instruments, Houston, TX. He is the leading author of three books: Active Noise Control Systems (New York: Wiley, 1996), Real-Time Digital Signal Processing (New York: Wiley, 2001), and Digital Signal Processors (Englewood Cliffs, NJ: Prentice-Hall, 2005). He holds seven U.S. patents, and has published over 150 technical papers. His research focuses on active noise and vibration control, real-time digital signal processing applications, adaptive echo and noise cancellation, digital audio applications, and digital communications.

Dr. Kuo received the IEEE Chester Sall Award for the first place paper (IEEE TRANSACTIONS ON CONSUMER ELECTRONICS) in 1993, and the faculty-of-year award in 2001 for accomplishments in research and scholarly areas. 